\title{
Delay-Dependent Robust Strictly Passive Analysis for a Class of Uncertain Discrete Singular Time-Delay Systems
}

\author{
Tianmin Huang*, Cuihong Wang ${ }^{\dagger}$ and Jianbin Qiu $^{\ddagger}$ \\ * School of Mathematics, Southwest Jiaotong University, Chengdu, 610031, P. R. China \\ email:tmhuangehome.swjtu.edu.cn \\ $\dagger$ School of Electrical Engineering, Southwest Jiaotong University, Chengdu, 610031, P. R. China \\ email:sxwangcuihong@yahoo.com.cn \\ $\ddagger$ Department of Manufacturing Engineering and Engineering Management \\ University of Science and Technology of China \& City University of Hong Kong Joint Advanced Research Center \\ Suzhou, 215123, P. R. China, email: jbqiu@mail.ustc.edu.cn
}

\begin{abstract}
The problem of robust strictly passive analysis for a class of uncertain discrete singular time-delay systems is investigated in this paper. The discrete singular systems under consideration involve constant time-delay and norm-bounded uncertainties. Based on an integral inequality, a new sufficient condition is firstly obtained, which guarantees that the discrete singular time-delay systems are admissible and strictly passive. Meanwhile, the sufficient condition for robust strictly passive is also obtained in terms of linear matrix inequality (LMI). A numerical example is also given to demonstrate the applicability of the proposed method.
\end{abstract}

\section{INTRODUCTION}

It is well known that dissipative theory plays an important role in stability analysis of control systems. The implication is that there exists a nonnegative energy function such that the energy consumption of a control system is always less that the supply rate of the energy. When the supply rate is taken as the special form, the dissipativeness is changed into passivity.

On the other hand, singular systems, which are also known as descriptor systems; semi-state space systems and generalized state space systems are dynamic systems whose behaviors are described by both differential equations (or difference equations) and algebraic equations. Such systems can preserve the structure of practical systems and have extensive applications in power systems, robotic systems and networks [1], [10]. The study of singular systems has received a lot of attention during the past decades, and many results based on the theory of state-space systems have been extended to the area of singular systems [2], [3], [4]. In recent years, the passive control for singular systems in terms of generalized algebraic Riccati inequalities has been proposed in [5]. [6] developed a linear matrix inequality (LMI) approach for designing the robust impulse passive state feedback controller and output feedback such that the closed-loop system is robust stable and strictly passive. In the singular time-delay systems setting, the passive control for continuous singular time-delay system can be found in [7], but for discrete singular time-delay system, there are few results involving the problem of positive real control.
In this paper, we study the problem of strictly passive analysis for discrete singular time-delay systems. Based on an integral inequality, a new sufficient condition ensuring a discrete singular time-delay system to be admissible and strictly passive is proposed. The obtained results are proposed in term of LMI and involves no decomposition of system matrices. Finally, an illustrative example is provided to demonstrate the applicability of the proposed method.

Notations: Throughout this paper, $\Re^{n}$ denotes the $n$-dimensional Euclidean space, $\Re^{n \times n}$ is the set of real matrices with $m$ rows and $n$ columns. For symmetric matrices $X$ and $Y$, the notation $X>Y$ (respectively, $X \geq Y$ ) means that $X-Y$ is positive definite (respectively, positive semidefinite). The superscripts $T$ and $*$ represent the transpose and the complex conjugate transpose, respectively. For a symmetric matrix, $\star$ denotes the matrix entries implied by symmetry. We use $\rho(E, A)$ to represent $\max \{|\lambda|: \operatorname{det}(\lambda E-$ $A)=0\} . \mathbf{I}_{n}$ and $\mathbf{0}_{m \times n}$ are used to denote the $n \times n$ identity matrix and $m \times n$ zero matrix, respectively. The subscripts $n$ and $m \times n$ are omitted when the size is not relevant or can be determined from the context.

\section{PROBlem Formulation}

Consider a class of uncertain discrete singular time-delay systems described by

$$
\left\{\begin{aligned}
E x(k+1)= & (A+\Delta A) x(k) \\
& +\left(A_{d}+\Delta A_{d}\right) x(k-d)+B_{w} w(k) \\
z(k)= & C x(k)+D_{w} w(k) \\
x(k)= & \phi(k), k \in[-d, 0]
\end{aligned}\right.
$$

where $x(k) \in \Re^{n}$ is the state; $w(k) \in \Re^{p}$ is the disturbance input and $z(k) \in \Re^{q}$ is the controlled output. For the above singular system, we assume that $p=q . d>0$ is an integer denoting the constant time-delay of system (1) and $\phi(k)$ is a real-valued vector function representing the initial condition of $x(k) . E, A, A_{d}, B_{w}, C$ and $D_{w}$ are constant matrices with appropriate dimensions, where $E$ may be singular and we assume that $\operatorname{rank}(E)=r \leq n . \Delta A, \Delta A_{d}$ are unknown and possibly time-varying matrices representing 
norm-bounded parameter uncertainties and are assumed to be of the following form

$$
\left[\begin{array}{cc}
\Delta A & \Delta A_{d}
\end{array}\right]=H F(k)\left[\begin{array}{ll}
N_{a} & N_{d}
\end{array}\right]
$$

where $H, N_{a}$ and $N_{d}$ are constant matrices with appropriate dimensions and $F(k)$ is an unknown matrix bounded by

$$
F^{T}(k) F(k) \leq \mathbf{I}
$$

Before proceeding further, we give some definitions and lemmas for the following nominal system.

$$
\left\{\begin{aligned}
E x(k+1) & =A x(k)+A_{d} x(k-d)+B_{w} w(k) \\
z(k) & =C x(k)+D_{w} w(k) \\
x(k) & =\phi(k), k \in[-d, 0]
\end{aligned}\right.
$$

\section{Definition 1.}

(1) The pair $(E, A)$ is said to be regular if $\operatorname{det}(z E-A)$ is not identically zero.

(2) The pair $(E, A)$ is said to be causal if $\operatorname{deg}(\operatorname{det}(z E-$ $A))=\operatorname{rank}(E)$.

(3) The pair $(E, A)$ is said to be stable if $\rho(E, A)<1$.

\section{Definition 2.}

(1) The discrete singular time-delay system (4) is said to be regular and causal, if the pair $(E, A)$ is regular and causal.

(2) The discrete singular time-delay system (4) is said to be admissible if it is regular, causal and stable.

Throughout this paper, we shall use the following concept of passiveness.

\section{Definition 3.}

(1) The discrete singular system (4) is said to be passive if there exists a positive definite functional $V(x(k))>0$ such that the following inequality holds

$$
2 \sum_{k=1}^{\infty} z^{T}(k) w(k) \geq 0
$$

for any disturbance input $\omega(k)$.

(2) The discrete singular system (4) is said to be strictly passive if there exists a positive definite functional $V(x(k))>0$ such that the following inequality holds

$$
2 \sum_{k=1}^{\infty} z^{T}(k) w(k)>0
$$

for any disturbance input $\omega(k)$.

Lemma 1. [8] Given matrices $X, Y$ and $\Omega=\Omega^{T}$, we have

$$
\Omega+X F(k) Y+Y^{T} F^{T}(k) X^{T}<0
$$

for any $F^{T}(k) F(k) \leq \mathbf{I}$ if and only if there exists a positive scalar $\varepsilon>0$ such that

$$
\Omega+\varepsilon^{-1} X X^{T}+\varepsilon Y^{T} Y<0 .
$$

Lemma 2. [9] Define $y(k):=x(k+1)-x(k)$, then for any matrices $M, N, Z \in \Re^{n \times n}$ and constant $d>0$, the following inequality holds:

$$
\begin{aligned}
& -\sum_{k-d}^{k-1} y^{T}(i) R y(i) \\
\leq & \xi^{T}(k)\left[\begin{array}{cc}
M^{T}+M & -M^{T}+N \\
* & -N^{T}-N
\end{array}\right] \xi(k) \\
& +d \xi^{T}(k)\left[\begin{array}{c}
M^{T} \\
N^{T}
\end{array}\right] Z^{-1}\left[\begin{array}{ll}
M & N
\end{array}\right] \xi(k) .
\end{aligned}
$$

\section{ROBUST PASSIVE ANALYSIS}

In this section, a sufficient condition which guarantees the system (4) to be admissible and strictly passive is proposed.

Theorem 1. For given time-delay $d>0$, the discrete singular time-delay system (4) is admissible and strictly passive if there exist matrices $P=P^{T}>0, Q=Q^{T}>0$, $Z=Z^{T}>0, S, M$ and $N$ such that the linear matrix inequality (5) shown on the top of next page hold, where

$\Xi_{11}:=A^{T} R S^{T}+S R^{T} A-E^{T} P E+M^{T} E+E^{T} M+Q$ and $R \in \Re^{n \times(n-r)}$ is any matrix with full column rank and satisfies $E^{T} R=0$.

Proof. By using Schur complement, it follows from (5) that

$$
\begin{aligned}
& \left(A+A_{d}\right)^{T} P\left(A+A_{d}\right)-E^{T} P E \\
& +\left(A+A_{d}\right)^{T} R S^{T}+S R^{T}\left(A+A_{d}\right)<0 .
\end{aligned}
$$

By denoting $U:=P E+R S^{T}$ and using the results in [10], it can be easily verified that the pair $\left(E, A+A_{d}\right)$ is admissible.

Note that $\operatorname{rank}(E) \leq n$, there exist two nonsingular matrices $\bar{G}$ and $\bar{H}$ such that

$$
\bar{E}=G E H=\left[\begin{array}{cc}
\mathbf{I}_{r} & \mathbf{0} \\
\mathbf{0} & \mathbf{0}
\end{array}\right] \text {. }
$$

Now, define the following matrices

$$
\begin{aligned}
& \bar{A}:=G A H=\left[\begin{array}{ll}
\bar{A}_{11} & \bar{A}_{12} \\
\bar{A}_{21} & \bar{A}_{22}
\end{array}\right], \\
& \bar{A}_{d}:=G A_{d} H=\left[\begin{array}{ll}
\bar{A}_{d 11} & \bar{A}_{d 12} \\
\bar{A}_{d 21} & \bar{A}_{d 22}
\end{array}\right], \\
& \bar{P}:=G^{-T} P G^{-1}=\left[\begin{array}{ll}
\bar{P}_{11} & \bar{P}_{12} \\
\bar{P}_{21} & \bar{P}_{22}
\end{array}\right], \\
& \bar{Q}:=G Q H=\left[\begin{array}{cc}
\bar{Q}_{11} & \bar{Q}_{12} \\
\bar{Q}_{21} & \bar{Q}_{22}
\end{array}\right], \\
& \bar{M}:=G^{-T} M H=\left[\begin{array}{ll}
\bar{M}_{11} & \bar{M}_{12} \\
\bar{M}_{21} & \bar{M}_{22}
\end{array}\right], \\
& \bar{N}:=G^{-T} N H=\left[\begin{array}{ll}
\bar{N}_{11} & \bar{N}_{12} \\
\bar{N}_{21} & \bar{N}_{22}
\end{array}\right], \\
& \bar{Z}:=G^{-T} M G^{-1}=\left[\begin{array}{ll}
\bar{Z}_{11} & \bar{Z}_{12} \\
\bar{Z}_{21} & \bar{Z}_{22}
\end{array}\right], \\
& \bar{R}:=G^{T} R=\left[\begin{array}{c}
\mathbf{0} \\
\bar{\Phi}
\end{array}\right], \bar{C}:=C H^{-1}=\left[\begin{array}{ll}
\bar{C}_{1} & \bar{C}_{2}
\end{array}\right] .
\end{aligned}
$$

Left-and right-multiplying $\Xi_{11}$ with $H^{T}$ and $H$ yields

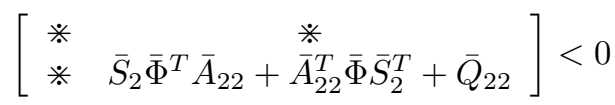




$$
\Xi:=\left[\begin{array}{cccccc}
\Xi_{11} & S R^{T} A_{d}+E^{T} N-M^{T} E & S R^{T} B_{w}-C^{T} & d M^{T} & A^{T} P & d(A-E)^{T} Z \\
\star & -N^{T} E-E^{T} N-Q & \mathbf{0} & d N^{T} & A_{d}^{T} P & d A_{d}^{T} Z \\
\star & \star & -D_{w}-D_{w}^{T} & \mathbf{0} & B_{w}^{T} P & d B_{w}^{T} Z \\
\star & \star & \star & -d Z & \mathbf{0} & \mathbf{0} \\
\star & \star & \star & \star & -P & \mathbf{0} \\
\star & \star & \star & \star & \star & -d Z
\end{array}\right]<0
$$

where * represents matrices that are not relevant in the following discussion. It follows from (7) that

$$
\bar{S}_{2} \bar{\Phi}^{T} \bar{A}_{22}+\bar{A}_{22}^{T} \bar{\Phi} \bar{S}_{2}^{T}+\bar{Q}_{22}<0
$$

Then, it can be easily shown that the matrix $\bar{S}_{2} \bar{\Phi}^{T} \bar{A}_{22}$ is nonsingular, which implies that the matrix $\bar{A}_{22}$ is nonsingular too. Then, we have that the pair $(E, A)$ is regular and causal.

Left-and right-multiplying (5) by

$$
\operatorname{diag}\left\{\bar{H}^{T}, \bar{H}^{T}, \mathbf{I}, \bar{G}^{-T}, \bar{G}^{-T}, \bar{G}^{-T}\right\}
$$

and

$$
\operatorname{diag}\left\{\bar{H}, \bar{H}, \mathbf{I}, \bar{G}^{-1}, \bar{G}^{-1}, \bar{G}^{-1}\right\}
$$

yields (9) shown on the top of next page, where

$\bar{\Xi}_{11}:=\bar{A}^{T} \bar{R} \bar{S}^{T}+\bar{S} \bar{R}^{T} \bar{A}-\bar{E}^{T} \bar{P} \bar{E}+\bar{M}^{T} \bar{E}+\bar{E}^{T} \bar{M}+\bar{Q}$ and $\bar{R} \in \Re^{n \times(n-r)}$ is any matrix with full column rank and satisfies $\bar{E}^{T} \bar{R}=0$.

Now, we define a transformation of state $x(k)$ as

$$
\bar{x}(k):=H^{-1} x(k)
$$

Then, the system (4) can be decomposed as

$$
\left\{\begin{aligned}
\bar{E} \bar{x}(k+1) & =\bar{A} \bar{x}(k)+\bar{A}_{d} \bar{x}(k-d)+\bar{B}_{w} w(k) \\
z(k) & =\bar{C} x(k)+D_{w} w(k)
\end{aligned}\right.
$$

Defining $\bar{y}(k):=\bar{x}(k+1)-\bar{x}(k)$ and considering system (11), we define the Lyapunov functional (12) shown on the next page.

Then, the forward difference of $V\left(\bar{x}_{k}\right)$ along the trajectory of system (11) is given by (13) shown on the next page.

In addition, it follows from that Lemma (2) that (14) shown on the next page is also true.

Furthermore, by noting $E^{T} R=0$, we can deduce (15) shown on the next page.

It follows from (12)-(15) that

$$
\Delta V\left(\bar{x}_{k}\right)-2 z^{T}(k) w(k) \leq \zeta^{T}(k) \bar{\Xi} \zeta(k) .
$$

Noting the zero initial condition of $x(k)$, we have $V(x(0))=0$. Then, it is easy to see that (9) guarantees

$$
\sum_{k=1}^{\infty}\left\{\Delta V(\bar{x}(k))-z^{T}(k) w(k)\right\}<0 .
$$

Therefore we can get

$$
\sum_{k=1}^{\infty} z^{T}(k) w(k)>V(x(\infty))-V(x(0)) .
$$

Noting the Definition 3, we have that the discrete singular time-delay system (4) is strictly passive.

Moreover, the LMI (9) obviously implies (18). Then, we can show that the singular system (4) with $w(k)=0$ is stable. This completes the proof.

In the following, we investigate the robust strictly passive analysis for discrete singular time-delay system (1).

Theorem 2. For given time-delay $d>0$, the discrete singular time-delay system (1) is admissible and strictly passive if there exist matrices $P=P^{T}>0, Q=Q^{T}>0$, $Z=Z^{T}>0, S, M, N$ and a positive scalar $\varepsilon>0$ such that the LMI (19) shown on the next page hold.

Proof. Replacing $A$ by $A+H F(k) N_{a}$ and $A_{d}$ by $A_{d}+$ $H F(k) N_{d}$ in (5) results in the following inequality:

$$
\Xi+X F(k) Y+Y^{T} F^{T}(k) X^{T}<0
$$

where $\Xi$ is defined in (5) and

$$
\begin{aligned}
X & :=\left[\begin{array}{lllllll}
H^{T} R S^{T} & \mathbf{0} & \mathbf{0} & \mathbf{0} & H^{T} P & H^{T} Z
\end{array}\right]^{T}, \\
Y & :=\left[\begin{array}{llllll}
N_{a} & N_{d} & \mathbf{0} & \mathbf{0} & \mathbf{0} & \mathbf{0}
\end{array}\right] .
\end{aligned}
$$

By using Lemma 1, it can be shown that (20) holds for any $F(k)$ satisfying (3) if and only if there exists a positive scalar $\varepsilon>0$ such that the following inequality holds:

$$
\Xi+\varepsilon^{-1} X X^{T}+\varepsilon Y^{T} Y<0
$$

which, by Shur Complement, is equivalent to (19). This completes the proof.

\section{NumERICAL EXAMPLE}

Consider the system (1) with parameters given in (22). By choosing $R=\left[\begin{array}{ll}0 & -1\end{array}\right]^{T}$ and using the LMI Toolbox in MATLAB to solve the feasibility problem of LMI (19), we obtain the solutions given in (23).

\section{CONCLUSION}

This paper has studied the problem of strictly passive analysis for discrete singular time-delay systems. A new sufficient condition ensuring that the system is admissible and strictly passive is obtained in terms of LMI. A numerical example is given to show the effectiveness of the proposed method.

\section{REFERENCES}

[1] L. Dai, Singular Control Systems, Berlin: Springer-Verlag, 1989.

[2] L.-Q. Zhang, J. Lam and S. Xu, "On positive realness of descriptor system," IEEE Transactions on Circuits and Systems-I, vol. 49, pp. 401-407, 2002.

[3] S. Xu and J. Lam, "New positive realness conditions for uncertain discrete descriptor systems: analysis and synthesis," IEEE Transactions on Circuits and Systems-I, vol. 51, pp. 1897-1905, 2004. 


$$
\bar{\Xi}:=\left[\begin{array}{cccccc}
\bar{\Xi}_{11} & \bar{S} \bar{R}^{T} \bar{A}_{d}+\bar{E}^{T} \bar{N}-\bar{M}^{T} \bar{E} & \bar{S} \bar{R}^{T} \bar{B}_{w}-\bar{C}^{T} & d \bar{M}^{T} & \bar{A}^{T} \bar{P} & d(\bar{A}-\bar{E})^{T} \bar{Z} \\
\star & -\bar{Q}-\bar{N}^{T} \bar{E}-\bar{E}^{T} \bar{N} & \mathbf{0} & d \bar{N}^{T} & \bar{A}_{d}^{T} \bar{P} & d \bar{A}_{d}^{T} \bar{Z} \\
\star & \star & -\bar{D}_{w}-\bar{D}_{w}^{T} & \mathbf{0} & \bar{B}_{w}^{T} \bar{P} & d \bar{B}_{w}^{T} \bar{Z} \\
\star & \star & \star & -d \bar{Z} & \mathbf{0} & \mathbf{0} \\
\star & \star & \star & \star & -\bar{P} & \mathbf{0} \\
\star & \star & \star & \star & \star & -d \bar{Z}
\end{array}\right]<0
$$

$$
\left\{\begin{aligned}
V\left(\bar{x}_{k}\right) & :=V_{1}(\bar{x}(k))+V_{2}(\bar{x}(k))+V_{3}(\bar{x}(k)) \\
V_{1}(\bar{x}(k)) & :=\bar{x}^{T}(k) \bar{E}^{T} \bar{P} \bar{E} \bar{x}(k) \\
V_{2}(\bar{x}(k)) & :=\sum_{i=1}^{d} \bar{x}^{T}(k-i) \bar{Q} \bar{x}(k-i) \\
V_{3}(\bar{x}(k)) & :=\sum_{i=-d+1}^{0} \sum_{j=-1+i}^{-1} \bar{y}^{T}(k+j) \bar{E}^{T} \bar{Z} \bar{E} \bar{y}(k+j)
\end{aligned}\right.
$$

$$
\left\{\begin{aligned}
\Delta V_{1}\left(\bar{x}_{k}\right) & =\bar{x}^{T}(k+1) \bar{E}^{T} \bar{P} \bar{E} \bar{x}(k+1)-\bar{x}^{T}(k) \bar{E}^{T} \bar{P} \bar{E} x(k), \\
\Delta V_{2}\left(\bar{x}_{k}\right) & =\bar{x}^{T}(k) \bar{Q} \bar{x}(k)-\bar{x}^{T}(k-d) \bar{Q} \bar{x}(k-d), \\
\Delta V_{3}(x(k)) & =d \bar{y}^{T}(k) \bar{E}^{T} \bar{Z} \bar{E} y(k)-\sum_{k-d}^{k-1} y^{T}(j) \bar{E}^{T} \bar{Z} \bar{E} y(j)
\end{aligned}\right.
$$

$$
\begin{aligned}
-\sum_{k-d}^{k-1} y^{T}(j) \bar{E}^{T} \bar{Z} \bar{E} y(j) \leq & \xi^{T}(k)\left[\begin{array}{cc}
\bar{M}^{T} \bar{E}+\bar{E}^{T} \bar{M} & -\bar{M}^{T} \bar{E}+\bar{E}^{T} \bar{N} \\
* & -\bar{N}^{T} \bar{E}-\bar{E}^{T} \bar{N}
\end{array}\right] \xi(k) \\
& +d \xi^{T}(k)\left[\begin{array}{c}
\bar{M}^{T} \\
\bar{N}^{T}
\end{array}\right] \bar{Z}^{-1}\left[\begin{array}{ll}
\bar{M} & \bar{N}
\end{array}\right] \xi(k)
\end{aligned}
$$

$$
0=2 \bar{x}^{T}(k+1) \bar{E}^{T} \bar{R} \bar{S} \bar{x}(k)=2 \bar{x}^{T}(k) \bar{A}^{T} \bar{R} \bar{S} \bar{x}(k)+2 \bar{x}^{T}(k-d) \bar{A}_{d}^{T} \bar{R} \bar{S} \bar{x}(k)+2 w^{T} \bar{B}_{w}^{T} \bar{R} \bar{S} \bar{x}(k)
$$

$$
\left[\begin{array}{ccccc}
\Xi_{11} & \bar{S} \bar{R}^{T} \bar{A}_{d}+\bar{E}^{T} \bar{N}-\bar{M}^{T} \bar{E} & d \bar{M}^{T} & \bar{A}^{T} \bar{P} & d(\bar{A}-\bar{E})^{T} \bar{Z} \\
\star & -\bar{Q}-\bar{N}^{T} \bar{E}-\bar{E}^{T} \bar{N} & d \bar{N}^{T} & \bar{A}_{d}^{T} \bar{P} & d \bar{A}_{d}^{T} \bar{Z} \\
\star & \star & \mathbf{0} & \bar{B}_{w}^{T} \bar{P} & d \bar{B}_{w}^{T} \bar{Z} \\
\star & \star & -d \bar{Z} & \mathbf{0} & \mathbf{0} \\
\star & \star & \star & -\bar{P} & \mathbf{0} \\
\star & \star & \star & \star & -d \bar{Z}
\end{array}\right]<0
$$

$$
\left[\begin{array}{cccccccc}
\Xi_{11} & S R^{T} A_{d}+E^{T} N-M^{T} E & S R^{T} B_{w}-C^{T} & d M^{T} & A^{T} P & d(A-E)^{T} Z & S R^{T} H & \varepsilon N_{a}^{T} \\
\star & -N^{T} E-E^{T} N-Q & \mathbf{0} & d N^{T} & A_{d}^{T} P & d A_{d}^{T} Z & \mathbf{0} & \varepsilon N_{d}^{T} \\
\star & \star & -D_{w}-D_{w}^{T} & \mathbf{0} & B_{w}^{T} P & d B_{w}^{T} Z & \mathbf{0} & \mathbf{0} \\
\star & \star & \star & -d Z & \mathbf{0} & \mathbf{0} & \mathbf{0} & \mathbf{0} \\
\star & \star & \star & \star & -P & \mathbf{0} & P^{T} H & \mathbf{0} \\
\star & \star & \star & \star & \star & -d Z & Z^{T} H & \mathbf{0} \\
\star & \star & \star & \star & \star & \star & -\varepsilon \mathbf{I} & \mathbf{0} \\
\star & \star & \star & \star & \star & \star & \star & -\varepsilon \mathbf{I}
\end{array}\right]<0
$$

[4] X. Ji, H. Su, and J. Chu, "An LMI approach to roubust stability of uncertain discrete singular time-delay systems," Asian Journal of Control, vol. 8, pp. 56-62.

[5] X. Dong, Q. Zhang, and L. Zhao, "Passive control of linear singular systems," Journal of Biomathematics, vol. 19, pp. 185-187, 2004.

[6] L. Yang, Q.-L. Zhang, X. Duan, D. Zhai, and G. Liu, "Robust passitive control of singular systems with uncertainties," in Proceedings of the 2006 American Control Conference, Minnesota, USA, 2006, pp. 3643- 


$$
\begin{gathered}
E=\left[\begin{array}{ll}
1 & 0 \\
0 & 0
\end{array}\right], A=\left[\begin{array}{cc}
0.6 & 0.25 \\
0.8 & 1
\end{array}\right], A_{d}=\left[\begin{array}{cc}
-0.5 & 0.3 \\
0.25 & -0.1
\end{array}\right], B=B_{w}=\left[\begin{array}{c}
0 \\
0.01
\end{array}\right] \\
H=\left[\begin{array}{l}
0.2 \\
0.2
\end{array}\right], N_{a}=N_{b}=\left[\begin{array}{ll}
0.2 & 0.2
\end{array}\right], D_{w}=1, C=\left[\begin{array}{ll}
1 & 0
\end{array}\right], D=0.8
\end{gathered}
$$

$$
\begin{gathered}
P=\left[\begin{array}{ll}
0.0034 & 0.0044 \\
0.0044 & 0.0307
\end{array}\right], Q=\left[\begin{array}{ll}
14.6297 & 15.3364 \\
15.3364 & 20.0098
\end{array}\right], Z=\left[\begin{array}{ll}
0.0468 & 0.1008 \\
0.1008 & 4.9824
\end{array}\right], \\
S=\left[\begin{array}{l}
-37.6281 \\
-45.9894
\end{array}\right], M=\left[\begin{array}{ll}
-0.0876 & 2.4613 \\
-0.1142 & 3.3939
\end{array}\right], N=\left[\begin{array}{cc}
-0.0913 & -1.6759 \\
-0.1061 & -2.6973
\end{array}\right], \varepsilon=80.9511, d=7 .
\end{gathered}
$$

3647.

[7] F. Chen, W.-D. Zhang and W. Wang, "Roubst passive control for uncertain singular system with state delay," in Proceeding of the 2006 Americal Control Conference, Minnesota, USA, 2006, pp. 14-16.

[8] I. R. Petersen, "A stabilization algorithm for a class of uncertain linear systems," Systems and Control Letters, vol. 8, pp. 351-357, 1987.
[9] X.-M. Zhang and M. Wu. "Delay-dependent $\mathcal{H}_{\infty}$ control for linear discrete-time uncertain systems with multiple unknown delays," Journal of Control Theory and Applications, vol. 23, pp. 918-922, 2002.

[10] S. Xu and J. Lam, Lecture notes in control and information on robust control and filtering of singular systems, Berlin:Springer-Verlag, 2006. 